\title{
Asymptotic behavior of Laplacian-energy-like invariant of the 3.6.24 lattice with various boundary conditions
}

\author{
Jia-Bao Liu' ${ }^{1}$, Jinde Cao ${ }^{2,3^{*}}$, Tasawar Hayat ${ }^{3}$ and Fuad E. Alsaadi ${ }^{3}$
}

*Correspondence:
jdcao@seu.edu.cn
2 Department
of Mathematics,
and Research Center
for Complex Systems
and Network Sciences,
Southeast University,
Nanjing 210096, People's
Republic of China
Full list of author information
is available at the end of the
article

*Correspondence: jdcao@seu.edu.cn of Mathematics, and Research Center for Complex Systems and Network Sciences, Nanjing 210096, People's Full list of author information article

\begin{abstract}
Let $G$ be a connected graph of order $n$ with Laplacian eigenvalues $\mu_{1}(G) \geq \mu_{2}(G) \geq \cdots \geq \mu_{n}(G)=0$. The Laplacian-energy-like invariant of $G$, is defined as $\mathscr{L} \mathscr{E} \mathscr{L}(G)=\sum_{i=1}^{n-1} \sqrt{\mu_{i}}$. In this paper, we investigate the asymptotic behavior of the 3.6.24 lattice in terms of Laplacian-energy-like invariant as $m, n$ approach infinity. Additionally, we derive that $M^{t}(n, m), M^{c}(n, m)$ and $M^{f}(n, m)$ have the same asymptotic Laplacian-energy-like invariants.
\end{abstract}

Keywords: Lattice, Toroidal lattice, Laplacian-energy-like invariant, Laplacian spectrum

\section{Background}

Throughout this paper, only undirected and simple connected graphs are considered. Let $G$ be a simple graph with $n$ vertices. The adjacency matrix $A=\left(a_{i j}\right)$ of $G$ is a $(0,1)$-square matrix of order $n$ whose $(i, j)$-entry is equal to 1 if $v_{i}$ is adjacent to $v_{j}$ and equal to 0 , otherwise. Let $D(G)=\operatorname{diag}\left(d_{1}, d_{2}, \ldots, d_{n}\right)$ be the diagonal matrix associated to $G$, where $d_{i}$ is the degree of vertex $v_{i}$. The matrix $L(G)=D(G)-A(G)$ is called Laplacian matrix of $G$. Let $M$ be a matrix representation of a graph $G$. For a graph $G$, let $M=M(G)$ be a corresponding graph matrix definned in a prescribed way. The M-polynomial of $G$ is defined as $\phi_{M}(G ; \lambda)=\operatorname{det}(\lambda I-M)$, where $I$ is the identity matrix. The M-eigenvalues of $G$ are the eigenvalues of $M$ together with their multiplicities. The $M$-spectrum of $G$ is the multiset of M-eigenvalues of $G$. In the case of the adjacency matrix (resp. Laplacian matrix), we simply refer to the A-eigenvalues (resp. L-eigenvalues) and A-spectrum (resp. L-spectrum) as the eigenvalues (resp. L-eigenvalues) of $G$. We denote the eigenvalues of $A(G)$, and $L(G)$ by $\lambda_{1}(G) \geq \lambda_{2}(G) \geq \cdots \geq \lambda_{n}(G)$, and $\mu_{1}(G) \geq \mu_{2}(G) \geq \cdots \geq \mu_{n}(G)=0$, respectively. Details on its theory can be found in recent papers (Wang 2014; Liu et al. 2014a, 2016c; Gao et al. 2012; Mohar and Alavi 1991; Liu and Pan 2015a) and the references cited therein.

For the connected graph $G$, the Laplacian-energy-like invariant of $G$ (Liu and Liu 2008), is defined as $\mathscr{L} \mathscr{E} \mathscr{L}(G)=\sum_{i=1}^{n-1} \sqrt{\mu_{i}}$. A general problem of interest in physics, chemistry and mathematics is the calculation of the Laplacian-energy-like invariant of graphs (Wang 2014; Liu et al. 2011), which has now become a popular topic of research.

(c) 2016 The Author(s). This article is distributed under the terms of the Creative Commons Attribution 4.0 International License (http://creativecommons.org/licenses/by/4.0/), which permits unrestricted use, distribution, and reproduction in any medium, provided you give appropriate credit to the original author(s) and the source, provide a link to the Creative Commons license, and indicate if changes were made. 
For more work on $\mathscr{L} \mathscr{E} \mathscr{L}(G)$, the readers are referred to the most recent papers (Liu and Pan 2015b; Liu et al. 2015, 2016ab, ; Das and Gutman 2014).

Historically in lattice statistics, the hexagonal lattice, 3.12.12 lattice and 3.6.24 lattice have attracted the most attention (Liu and Yan 2013; Ye 2011b; Zhang 2013). Some topological indices of graphs were studied in Li et al. (2015), Yan and Zhang (2009), Ye (2011a), Liu et al. (2014b, 2016d) and Liu and Pan (2016). In fact, Liu et al. have already studied the asymptotic incidence energy (Liu and Pan 2015a) and the Laplacian-energylike invariant of lattices (Liu et al. 2015).

It is an interesting problem to study the various energies of some lattices with various boundary conditions. W. Wang considered the behavior of Laplacian-energy-like invariant of some graphs in Wang (2014). In present paper, we derive the the Laplacianenergy-like invariant of 3.6.24 lattice via the graph spectrum of the line graph of the subdivision graph of a graph $G$ with the help of computer calculation, which is different from the approach of Wang (2014). Yan et al. investigated the asymptotic behavior of some indices of iterated line graphs of regular graphs in Liu et al. (2016c). Motivated by the above results, in this paper we consider the problem of computations of the $\mathscr{L} \mathscr{E} \mathscr{L}(G)$ of the 3.6.24 lattice with various boundary conditions.

\section{Preliminaries}

We first recall some underlying definitions and lemmas in graph theory.

\section{Some definitions and lemmas}

The subdivision graph $s(G)$ of a graph $G$ is obtained from $G$ by deleting every edge $u v$ of $G$ and replacing it by a vertex $w$ of degree 2 that is joined to $u$ and $v$ (see p. 151 of Chartrand and Zhang 2004).

The line graph of a graph $G$, denoted by $l(G)$, is the graph whose vertices correspond to the edges of $G$ with two vertices of $l(G)$ being adjacent if and only if the corresponding edges in $G$ share a common vertex (Klein and Yi 2012).

Lemma 1 (Gao et al. 2012) Let $G$ be an $r$-regular connected graph with $n$ vertices and m edges, then

$$
\begin{aligned}
& \phi_{L}(l(G) ; x)=(x-2 r)^{m-n} \phi_{L}(G ; x), \\
& \phi_{L}(s(G) ; x)=(-1)^{m}(2-x)^{m-n} \phi_{L}(G ; x(r+2-x)),
\end{aligned}
$$

where $\phi_{L}(l(G) ; x)$ and $\phi_{L}(s(G) ; x)$ are the characteristic polynomial for the Laplacian matrix of graphs $l(G)$ and $s(G)$, respectively.

Let a bipartite graph $G$ with a bipartition $V(G)=(U, V)$ is called an $(r, s)$-semiregular graph if all vertices in $U$ have degree $r$ and all vertices in $V$ have degree $s$.

Lemma 2 (Mohar and Alavi 1991) Let $G$ be an $(r, s)$-semiregular connected graph with $n$ vertices. Then

$$
\phi_{L}(l(G) ; x)=(-1)^{n}(x-(r+s))^{m-n} \phi_{L}(G ;(r+s-x)),
$$


where $\phi_{L}(l(G) ; x)$ is the Laplacian characteristic polynomial of the line graph $l(G)$ and $m$ is the number of edges of $G$.

The 3.12.12 and 3.6.24 lattices

The 3.12.12 lattice with toroidal boundary condition (Liu and Yan 2013), denoted $J^{t}(n, m)$, is illustrated in Fig. 1. Many problems related to the 3.12.12 lattice were considered by physicists (Liu and Yan 2013; Zhang 2013; Liu et al. 2014b). The 3.6.24 lattice with toroidal boundary condition (Zhang 2013), denoted $M^{t}(n, m)$, is illustrated in Fig. 2.

Based on the constructions of the 3-12-12 and 3.6.24 lattices, we notice that a very important and interesting relationship between 3-12-12 lattice $J^{t}(n, m)$ and 3-6-24 $M^{t}(n, m)$ lattice. The relationship is illustrated as follows.

$$
\begin{array}{llll}
J^{t}(n, m) & \overrightarrow{s\left(J^{t}(n, m)\right)} & s\left(J^{t}(n, m)\right) & \overrightarrow{l\left(s\left(J^{t}(n, m)\right)\right)} \\
M^{t}(n ; m) &
\end{array}
$$

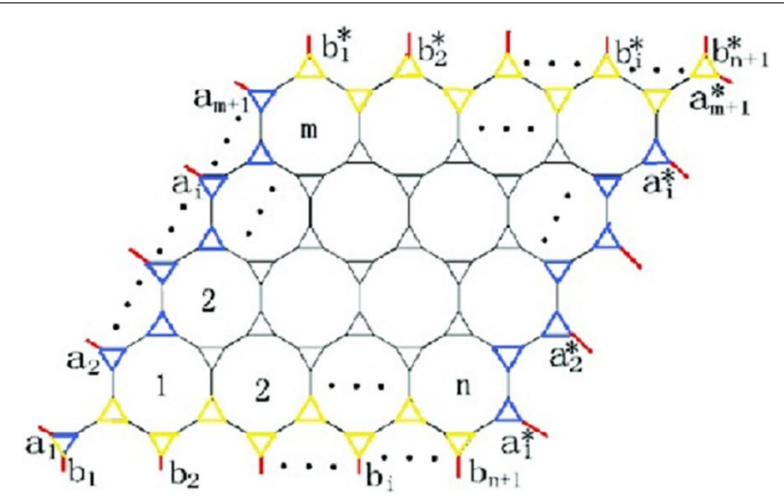

Fig. 1 The 3.12 .12 lattice $J^{t}(n, m)$ lattice with toroidal boundary condition

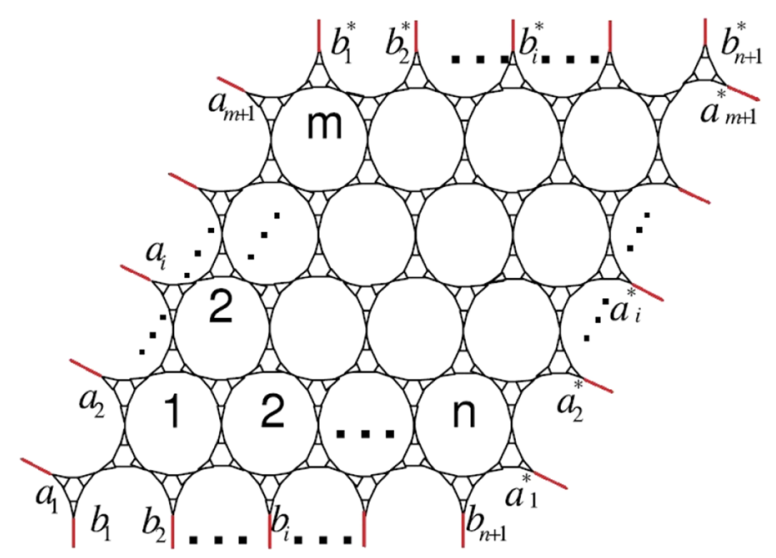

Fig. 2 The 3.6.24 lattice $M^{t}(n, m)$ lattice with toroidal boundary condition 


\section{Main results}

In this section, we will explore the Laplacian spectrum of the 3.6.24 lattice with toroidal boundary condition. We begin with the adjacency spectrum of 3.12 .12 lattice.

The following adjacency spectrum of 3.12.12 lattice is shown in Liu and Yan (2013).

Theorem 1 (Liu and Yan 2013) Let $J^{t}(n, m)$ be the 3.12.12 lattice with toroidal boundary condition. Then the adjacency spectrum is

$$
\begin{aligned}
& \operatorname{Spec}_{A}\left(J^{t}(n, m)\right)=\{\underbrace{-2,-2, \ldots,-2}_{(m+1)(n+1)}, \underbrace{0,0, \ldots, 0}_{(m+1)(n+1)}\} \\
& \bigcup\left\{\frac{1 \pm \sqrt{13 \pm 4 \sqrt{3+2 \cos \alpha_{i}+2 \cos \beta_{j}+2 \cos \left(\alpha_{i}+\beta_{j}\right)}}}{2}\right\},
\end{aligned}
$$

where $\alpha_{i}=\frac{2 \pi i}{m+1}, \beta_{j}=\frac{2 \pi j}{n+1}, i=0,1, \ldots, m ; j=0,1, \ldots, n$.

The Laplacian spectrum of the 3.12.12 lattice with toroidal boundary condition is given by the following theorem.

Theorem 2 Let $J^{t}(n, m)$ be the 3.12.12 lattice with toroidal boundary condition and $\alpha_{i}=\frac{2 \pi i}{m+1}, \beta_{j}=\frac{2 \pi j}{n+1}, i=0,1, \ldots, m ; j=0,1, \ldots, n$. Then the Laplacian spectrum is

$$
\begin{aligned}
& \operatorname{Spec}_{L}\left(J^{t}(n, m)\right)=\{\underbrace{3,3, \ldots, 3}_{(m+1)(n+1)}, \underbrace{5,5, \ldots, 5}_{(m+1)(n+1)}\} \\
& \bigcup\left\{\frac{5 \pm \sqrt{13 \pm 4 \sqrt{3+2 \cos \alpha_{i}+2 \cos \beta_{j}+2 \cos \left(\alpha_{i}+\beta_{j}\right)}}}{2}\right\} .
\end{aligned}
$$

Proof Consider that $J^{t}(n, m)$ is a 3-regular graph of order $n$, then $D(G)=3 I_{n}$. Hence,

$$
L\left(J^{t}(n, m)\right)=3 I_{n}-A\left(J^{t}(n, m)\right) .
$$

Define the map $\varphi\left(\lambda_{i}\right)=3-\lambda_{i}$ maps the eigenvalues of $A\left(J^{t}(n, m)\right)$ to the eigenvalues of $L\left(J^{t}(n, m)\right)$ and can be considered as an isomorphism of the $A$-spectrum to the corresponding the $L$-spectrum for $J^{t}(n, m)$. Based on the fact that $G$ is an $r$-regular graph with $n$ vertices and $\operatorname{Spec}_{A}(G)=\left\{\lambda_{1}, \lambda_{2}, \ldots, \lambda_{n}\right\}$.

Then $\operatorname{Spec}_{L}(G)=\left\{r-\lambda_{1}, r-\lambda_{2}, \ldots, r-\lambda_{n}\right\}$.

Next, we will deduce the Laplacian spectrum of the 3.6.24 lattice $M^{t}(n, m)$.

Theorem 3 Let $\mu_{1} \geq \mu_{2} \ldots \geq \mu_{6(m+1)(n+1)}=0$ are the Laplacian eigenvalues of the 3.12.12 lattice $J^{t}(n, m)$. Then the Laplacian spectrum of $M^{t}(n, m)$ is 


$$
\begin{gathered}
\operatorname{Spec}_{L}\left(M^{t}(n, m)\right)=\{\underbrace{3,3, \ldots, 3}_{3(m+1)(n+1)}, \underbrace{5,5, \ldots, 5}_{3(m+1)(n+1)}\} \\
\bigcup_{i=1}^{6(m+1)(n+1)}\left\{\frac{5-\sqrt{25-4 \mu_{i}}}{2}\right\} \\
\bigcup_{i=1}^{6(m+1)(n+1)}\left\{\frac{5+\sqrt{25-4 \mu_{i}}}{2}\right\} .
\end{gathered}
$$

Proof Note that $J^{t}(n, m)$ has $6(m+1)(n+1)$ vertices and $M^{t}(n, m)$ is the line graph of the subdivision of $J^{t}(n, m)$ which is a 3 -regular graph. That is,

$$
M^{t}(n, m)=l\left(s\left(J^{t}(n, m)\right)\right)
$$

For the convenience of description, we suppose that $s\left(J^{t}(n, m)\right)$ has $p$ vertices and $q$ edges. Obviously, $p=15(m+1)(n+1)$ and $q=18(m+1)(n+1)$, respectively. In fact, $s\left(J^{t}(n, m)\right)$ is $(2,3)$-semi-regular graphs.

By Lemma 2, suppose the graph in equality above is $s\left(J^{t}(n, m)\right)$, then the Laplacian characteristic polynomial of $l\left(s\left(J^{t}(n, m)\right)\right)$ is,

$$
\begin{aligned}
\phi_{L}\left(l\left(s\left(J^{t}(n, m)\right)\right) ; x\right)= & (-1)^{p}(x-5)^{q-p} \\
& \times \phi_{L}\left(s\left(J^{t}(n, m)\right) ;(5-x)\right),
\end{aligned}
$$

By virtue of Eq. (1), one can immediately obtain that

$$
\begin{aligned}
\phi_{L}\left(M^{t}(n, m) ; x\right)= & (-1)^{p}(x-5)^{q-p} \\
& \times \phi_{L}\left(s\left(J^{t}(n, m)\right) ;(5-x)\right) .
\end{aligned}
$$

On the other hand, note that $J^{t}(n, m)$ has $9(m+1)(n+1)$ edges, it obviously follows from Lemma 1,

$$
\begin{aligned}
\phi_{L}\left(s\left(J^{t}(n, m)\right) ; x\right)= & (-1)^{9(m+1)(n+1)}(2-x)^{3(m+1)(n+1)} \\
& \times \phi_{L}\left(J^{t}(n, m) ;(x(5-x))\right) .
\end{aligned}
$$

Consider the term $\phi_{L}\left(s\left(J^{t}(n, m)\right) ;(5-x)\right)$ in Eq. (3), we replace $x$ with $5-x$ in Eq. (4), we have

$$
\begin{aligned}
\phi_{L}\left(s\left(J^{t}(n, m)\right) ;(5-x)\right)= & (-1)^{9(m+1)(n+1)}(x-3)^{3(m+1)(n+1)} \\
& \times \phi_{L}\left(J^{t}(n, m) ;(x(5-x))\right) .
\end{aligned}
$$


Combing Eq. (3) with Eq. (5), $p=15(m+1)(n+1)$ and $q=18(m+1)(n+1)$, it holds

$$
\begin{aligned}
\phi_{L}\left(M^{t}(n, m) ; x\right)= & (-1)^{p+9(m+1)(n+1)}(x-5)^{q-p} \\
& \times(x-3)^{3(m+1)(n+1)} \phi_{L}\left(J^{t}(n, m) ;(x(5-x))\right) \\
= & (-1)^{24(m+1)(n+1)}((x-3)(x-5))^{3(m+1)(n+1)} \\
& \times \phi_{L}\left(J^{t}(n, m) ;(x(5-x))\right) .
\end{aligned}
$$

Note that the roots of $x(5-x)=\mu_{i}$ are

$$
x_{1, i}=\frac{5-\sqrt{25-4 \mu_{i}}}{2}, \quad x_{2, i}=\frac{5+\sqrt{25-4 \mu_{i}}}{2},
$$

where $\mu_{1} \geq \mu_{2} \ldots \geq \mu_{6(m+1)(n+1)}=0$ are the Laplacian eigenvalues of the 3.12.12 lattice $J^{t}(m, n)$.

It follows from Eq. (6) that the Laplacian spectrum of $M^{t}(n, m)$ is

$$
\begin{aligned}
& \operatorname{spec}_{L}\left(M^{t}(n, m)\right)=\{\underbrace{3,3, \ldots, 3}_{3(m+1)(n+1)}, \underbrace{5,5, \ldots, 5}_{3(m+1)(n+1)}\} \\
& \bigcup_{i=1}^{6(m+1)(n+1)}\left\{\frac{5-\sqrt{25-4 \mu_{i}}}{2}\right\} \bigcup_{i=1}^{6(m+1)(n+1)}\left\{\frac{5+\sqrt{25-4 \mu_{i}}}{2}\right\},
\end{aligned}
$$

where $\mu_{i}$ are the Laplacian eigenvalues of the 3.12.12 lattice $J^{t}(n, m)$.

Theorem 4 Let $A=$

$$
\sqrt{5-\sqrt{15 \pm 2 \sqrt{13 \pm 4 \sqrt{3+2 \cos \alpha_{i}+2 \cos \beta_{j}+2 \cos \left(\alpha_{i}+\beta_{j}\right)}}}}
$$

$B=$

$$
\sqrt{5+\sqrt{15 \pm 2 \sqrt{13 \pm 4 \sqrt{3+2 \cos \alpha_{i}+2 \cos \beta_{j}+2 \cos \left(\alpha_{i}+\beta_{j}\right)}}}}
$$

and $\alpha_{i}=\frac{2 \pi i}{m+1}, \beta_{j}=\frac{2 \pi j}{n+1}, i=0,1, \ldots, m ; j=0,1, \ldots, n$. Then

1. The Laplacian-energy-like invariant of $M^{t}(n, m)$ can be expressed as

$$
\begin{aligned}
& \mathscr{L} \mathscr{E} \mathscr{L}\left(M^{t}(n, m)\right)=3(\sqrt{3}+\sqrt{5})(m+1)(n+1) \\
& +\frac{(m+1)(n+1)}{\sqrt{2}}(\sqrt{5-\sqrt{5}}+\sqrt{5-\sqrt{13}}) \\
& +\frac{(m+1)(n+1)}{\sqrt{2}}(\sqrt{5+\sqrt{5}}+\sqrt{5+\sqrt{13}}) \\
& +\frac{1}{\sqrt{2}} \sum_{i=0}^{m} \sum_{j=0}^{n} A+\frac{1}{\sqrt{2}} \sum_{i=0}^{m} \sum_{j=0}^{n} B \text {. }
\end{aligned}
$$


2. $\mathscr{L} \mathscr{E} \mathscr{L}\left(M^{t}(n, m)\right) \approx 18.1764(m+1)(n+1)$, as $m, n \rightarrow \infty$.

Proof Based on Theorems 2, 3 and the definition of the Laplacian-energy-like invariant, we can arrive at the statement 1 of Theorem 4 .

Note that the term $A$ can decompose four terms

$$
\begin{aligned}
& A=A_{1}+A_{2}+A_{3}+A_{4} \text {, } \\
& A_{1}=\sqrt{5-\sqrt{15-2 \sqrt{13-4 \sqrt{3+2 \cos \alpha_{i}+2 \cos \beta_{j}+2 \cos \left(\alpha_{i}+\beta_{j}\right)}}}}, \\
& A_{2}=\sqrt{5-\sqrt{15-2 \sqrt{13+4 \sqrt{3+2 \cos \alpha_{i}+2 \cos \beta_{j}+2 \cos \left(\alpha_{i}+\beta_{j}\right)}}}}, \\
& A_{3}=\sqrt{5-\sqrt{15+2 \sqrt{13-4 \sqrt{3+2 \cos \alpha_{i}+2 \cos \beta_{j}+2 \cos \left(\alpha_{i}+\beta_{j}\right)}}}}, \\
& A_{4}=\sqrt{5-\sqrt{15+2 \sqrt{13+4 \sqrt{3+2 \cos \alpha_{i}+2 \cos \beta_{j}+2 \cos \left(\alpha_{i}+\beta_{j}\right)}}} .}
\end{aligned}
$$

Similarly,

$$
\begin{aligned}
& B=B_{1}+B_{2}+B_{3}+B_{4}, \\
& B_{1}=\sqrt{5+\sqrt{15-2 \sqrt{13-4 \sqrt{3+2 \cos \alpha_{i}+2 \cos \beta_{j}+2 \cos \left(\alpha_{i}+\beta_{j}\right)}}}} \\
& B_{2}=\sqrt{5+\sqrt{15-2 \sqrt{13+4 \sqrt{3+2 \cos \alpha_{i}+2 \cos \beta_{j}+2 \cos \left(\alpha_{i}+\beta_{j}\right)}}}} \\
& B_{3}=\sqrt{5+\sqrt{15+2 \sqrt{13-4 \sqrt{3+2 \cos \alpha_{i}+2 \cos \beta_{j}+2 \cos \left(\alpha_{i}+\beta_{j}\right)}}}} \\
& B_{4}=\sqrt{5+\sqrt{15+2 \sqrt{13+4 \sqrt{3+2 \cos \alpha_{i}+2 \cos \beta_{j}+2 \cos \left(\alpha_{i}+\beta_{j}\right)}}}} .
\end{aligned}
$$


Then

$$
\begin{aligned}
& \lim _{m \rightarrow \infty} \lim _{n \rightarrow \infty} \frac{\mathscr{L} \mathscr{E} \mathscr{L}\left(M^{t}(m, n)\right)}{18(m+1)(n+1)}=\frac{\sqrt{3}+\sqrt{5}}{6} \\
& +\frac{\sqrt{2}}{36}(\sqrt{5-\sqrt{5}}+\sqrt{5-\sqrt{13}}+\sqrt{5+\sqrt{5}}+\sqrt{5+\sqrt{13}}) \\
& +\lim _{m \rightarrow \infty} \lim _{n \rightarrow \infty} \frac{\sqrt{2}}{36(m+1)(n+1)} \sum_{i=0}^{m} \sum_{j=0}^{n}\left(A_{1}+A_{2}+A_{3}+A_{4}\right) \\
& +\lim _{m \rightarrow \infty} \lim _{n \rightarrow \infty} \frac{\sqrt{2}}{36(m+1)(n+1)} \sum_{i=0}^{m} \sum_{j=0}^{n}\left(B_{1}+B_{2}+B_{3}+B_{4}\right) .
\end{aligned}
$$

We consider that

$$
\begin{aligned}
\lim _{m \rightarrow \infty} \lim _{n \rightarrow \infty} \frac{\sqrt{2}}{36(m+1)(n+1)} \sum_{i=0}^{m} \sum_{j=0}^{n}\left(A_{1}+A_{2}+A_{3}+A_{4}\right) \\
=\frac{\sqrt{2}}{36} \cdot \frac{1}{4 \pi^{2}} \int_{0}^{2 \pi} \int_{0}^{2 \pi} A_{1}^{\prime} d x d y \\
+\frac{\sqrt{2}}{36} \cdot \frac{1}{4 \pi^{2}} \int_{0}^{2 \pi} \int_{0}^{2 \pi} A_{2}^{\prime} d x d y \\
+\frac{\sqrt{2}}{36} \cdot \frac{1}{4 \pi^{2}} \int_{0}^{2 \pi} \int_{0}^{2 \pi} A_{3}^{\prime} d x d y \\
+\frac{\sqrt{2}}{36} \cdot \frac{1}{4 \pi^{2}} \int_{0}^{2 \pi} \int_{0}^{2 \pi} A_{4}^{\prime} d x d y \\
\approx 0.0040,
\end{aligned}
$$

where

$$
\begin{aligned}
& A_{1}^{\prime}=\sqrt{5-\sqrt{15-2 \sqrt{13-4 \sqrt{3+2 \cos x+2 \cos y+2 \cos (x+y)}}}} \\
& A_{2}^{\prime}=\sqrt{5-\sqrt{15-2 \sqrt{13+4 \sqrt{3+2 \cos x+2 \cos y+2 \cos (x+y)}}}} \\
& A_{3}^{\prime}=\sqrt{5-\sqrt{15+2 \sqrt{13-4 \sqrt{3+2 \cos x+2 \cos y+2 \cos (x+y)}}}} \\
& A_{4}^{\prime}=\sqrt{5-\sqrt{15+2 \sqrt{13+4 \sqrt{3+2 \cos x+2 \cos y+2 \cos (x+y)}}}} .
\end{aligned}
$$

The above numerical integration values are calculated by using the computer software Matlab. 
By a complectly similar calculation with software Matlab, we can obtain that

$$
\begin{aligned}
& \lim _{m \rightarrow \infty} \lim _{n \rightarrow \infty} \frac{\sqrt{2}}{36(m+1)(n+1)} \sum_{i=0}^{m} \sum_{j=0}^{n}\left(B_{1}+B_{2}+B_{3}+B_{4}\right) \\
& \approx 0.0118 .
\end{aligned}
$$

Consequently, we have

$$
\begin{aligned}
\lim _{m \rightarrow \infty} & \lim _{n \rightarrow \infty} \frac{\mathscr{L} \mathscr{E}\left(M^{t}(n, m)\right)}{18(m+1)(n+1)} \\
\approx & \frac{\sqrt{3}+\sqrt{5}}{6}+\frac{\sqrt{2}}{36}(\sqrt{5-\sqrt{5}}+\sqrt{5-\sqrt{13}} \\
& +\sqrt{5+\sqrt{5}}+\sqrt{5+\sqrt{13}}) \\
& +0.0040+0.0118 \\
= & 1.0098 .
\end{aligned}
$$

The Eq. (7) implies $M^{t}(n, m)$ has the asymptotic Laplacian-energy-like invariant

$$
\mathscr{L} \mathscr{E} \mathscr{L}\left(M^{t}(n, m)\right) \approx 18.1764(m+1)(n+1)
$$

as $m, n \rightarrow \infty$. The theorem thus follows.

The energy of a graph $G$ with $n$ vertices, denoted by $\mathscr{E}(G)$, is defined by

$$
\mathscr{E}(G)=\sum_{i=1}^{n}\left|\lambda_{i}(G)\right|
$$

where the $\lambda_{i}(G)$ are the eigenvalues of the adjacency matrix of $G$. The asymptotic energy per vertex of $G$ (Yan and Zhang 2009) is defined by

$$
\lim _{n \rightarrow \infty} \frac{\mathscr{E}(G)}{\left|V\left(G_{n}\right)\right|}
$$

Motivated by the above results, we consider the problem of computation of the $\mathscr{L} \mathscr{E} \mathscr{L}(G)$ per vertex of $G$ (Liu et al. 2015).

Theorem 5 (Liu et al. 2015) Let $\left\{G_{n}\right\}$ be a sequence of finite simple graphs with bounded average degree such that

$$
\lim _{n \rightarrow \infty}\left|V\left(G_{n}\right)\right|=\infty, \quad \lim _{n \rightarrow \infty} \frac{\mathscr{L} \mathscr{E} \mathscr{L}\left(G_{n}\right)}{\left|V\left(G_{n}\right)\right|}=h \neq 0 .
$$

Let $\left\{H_{n}\right\}$ be a sequence of spanning subgraphs of $\left\{G_{n}\right\}$ such that

$$
\lim _{n \rightarrow \infty} \frac{\left|v \in V\left(H_{n}\right): d_{H_{n}(v)}=d_{G_{n}(v)}\right|}{\left|V\left(G_{n}\right)\right|}=1,
$$


then

$$
\lim _{n \rightarrow \infty} \frac{\mathscr{L} \mathscr{E} \mathscr{L}\left(H_{n}\right)}{\left|V\left(G_{n}\right)\right|}=h .
$$

That is, $G_{n}$ and $H_{n}$ have the same asymptotic Laplacian-energy-like invariant.

Remark 1 Theorem 5 provides a very effective approach to handle the asymptotic the Laplacian-energy-like invariant of a graph with bounded average degree.

Based on Theorem 5, the following result is straightforward.

Theorem 6 Let $M^{t}(n, m)$ (resp. $\left.M^{c}(n, m), M^{f}(n, m)\right)$ be the toroidal (resp. cylindrical, free) boundary condition of the 3.6.24 lattice. Then

$$
\begin{aligned}
\mathscr{L} \mathscr{E} \mathscr{L}\left(M^{t}(n, m)\right) \\
=\mathscr{L} \mathscr{E} \mathscr{L}\left(M^{c}(n, m)\right) \\
=\mathscr{L} \mathscr{E} \mathscr{L}\left(M^{f}(n, m)\right) \\
\approx 18.1764(m+1)(n+1) .
\end{aligned}
$$

Remark 2 It follows from Theorems 5 and 6 that the growth rate of the $\mathscr{L} \mathscr{E} \mathscr{L}(G)$ of the 3.6.24 lattice $M^{t}(n, m)$ (resp. $\left.M^{c}(n, m), M^{f}(n, m)\right)$ with toroidal (resp. cylindrical, free) boundary condition is only dependent on the number of vertices of it.

\section{Conclusions}

In this paper, we deduced the formulae expressing the Laplacian-energy-like invariant of the 3.6.24 lattice with various boundary conditions. Moreover, we obtained the explicit asymptotic values of the Laplacian-energy-like invariant by utilizing the analysis methods with the help of software Matlab calculation. In addition, we showed that their growth rates are independent of the structure of $M(n, m)$ and only dependent on the number of vertices of $M(n, m)$. These and some other related issues are very good topics on lattices, which deserves further exploration.

Authors' contributions

J-BL designed the study and completed the article. JC provided the study design. TH and FEA provided scientific advice. All authors read and approved the final manuscript.

\section{Author details}

${ }^{1}$ School of Mathematics and Physics, Anhui Jianzhu University, Hefei 230601, People's Republic of China. ${ }^{2}$ Department of Mathematics, and Research Center for Complex Systems and Network Sciences, Southeast University, Nanjing 210096, People's Republic of China. ${ }^{3}$ Nonlinear Analysis and Applied Mathematics (NAAM) Research Group, Department of Mathematics, Faculty of Science, King Abdulaziz University, Jeddah 21589, Saudi Arabia.

\section{Acknowledgements}

The authors would like to express their sincere gratitude to the anonymous referees for many friendly and helpful suggestions, which led to great deal of improvement of the original manuscript. This work is supported by the National Natural Science Foundation of China (grant 11601006, 71571002). 
Received: 21 August 2015 Accepted: 9 August 2016

Published online: 24 August 2016

\section{References}

Chartrand G, Zhang P (2004) Introduction to graph theory. McGraw-Hill, Kalamazoo, MI

Das KC, Gutman I (2014) On Laplacian-energy-like invariant of graphs. Linear Algebra Appl 446:329-344

Gao X, Luo Y, Liu W (2012) Kirchhoff index in line, subdivision and total graphs of a regular graph. Dicrete Appl Math $160: 560$

Klein DJ, Yi E (2012) A comparison on metric dimension of graphs, line graphs, and line graphs of the subdivision graphs. Eur J Pure Appl Math 5(3):302-316

Liu B, Huang Y, You Z (2011) A survey on the Laplacian-energy-like invariant. MATCH Commun Math Comput Chem $66: 713-730$

Liu JB, Cao J, Xie J (2014a) On the incidence energy of some toroidal lattices. Abstr Appl Anal. Article ID 568153, pp 1-6

Liu JB, Pan XF, Cao J, Hu FF (2014b) A note on 'Some physical and chemical indices of clique-inserted lattices'. J Stat Mech Theory Exp 6(P06006):1-9

Liu JB, Pan XF, Hu FT, Hu FF (2015) Asymptotic Laplacian-energy-like invariant of lattices. Appl Math Comput 253:205-214

Liu JB, Cao J, Alofi A, AL-Mazrooei A, Elaiw A (2016a) Applications of Laplacian spectra for $n$-prism networks. Neurocomputing 198:69-73

Liu JB, Pan XF, Yu L, Li D (2016b) Complete characterization of bicyclic graphs with minimal Kirchhoff index. Discret Appl Math 200:95-107

Liu JB, Pan XF, Hu FT (2016c) The laplacian polynomial of graphs derived from regular graphs and applications. Ars Comb 126:289-300

Liu JB, Wang WR, Zhang YM, Pan XF (2016d) On degree resistance distance of cacti. Discrete Appl Math 203:217-225

Liu J, Liu B (2008) A Laplacian-energy-like invariant of a graph. MATCH Commun Math Comput Chem 59:355-372

Liu JB, Pan XF (2015a) Asymptotic incidence energy of lattices. Phys A 422:193-202

Liu JB, Pan XF (2015b) A unified approach to the asymptotic topological indices of various lattices. Appl Math Comput 270:62-73

Liu JB, Pan XF (2016) Minimizing Kirchhoff index among graphs with a given vertex bipartiteness. Appl Math Comput 291:84-88

Liu XY, Yan WG (2013) The triangular kagomé lattices revisited. Phys A 392:5615-5621

Li S, Yan WG, Tian T (2015) Some physical and chemical indices of the Union Jack lattice. J Stat Mech Theory Exp. P02014

Mohar B, Alavi Y (1991) The Laplacian spectrum of graphs. Graph Theory Comb Appl 2:871-898

Wang W (2014) Asymptotic behavior of Laplacian-energy-like invariant of some graphs. Ars Comb 115:273-282

Yan WG, Zhang ZH (2009) Asymptotic energy of lattices. Phys A 388:1463-1471

Ye L (2011a) The energy of a type of lattice. Appl Math Lett 24:145-148

Ye L (2011 b) On the Kirchhoff index of some toroidal lattices. Linear Multilinear Algebra 59:645-650

Zhang Z (2013) Some physical and chemical indices of clique-inserted lattices. J Stat Mech Theory Exp P10004

\section{Submit your manuscript to a SpringerOpen ${ }^{\circ}$ journal and benefit from:}

- Convenient online submission

- Rigorous peer review

- Immediate publication on acceptance

- Open access: articles freely available online

- High visibility within the field

- Retaining the copyright to your article

Submit your next manuscript at springeropen.com 